\section{Dupuytren's disease: what dermatologists need to know*}

Camila Ribeiro Amadio ${ }^{1}$

Barbara Machado Magalhães ${ }^{1}$

Felipe Yazawa ${ }^{1}$

Karine Simone ${ }^{2}$

DOI: http:/ / dx.doi.org/10.1590/abd1806-4841.20188035

Dear Editor,

Dupuytren's disease is a benign disorder of autosomal dominant inheritance and variable penetrance involving the gene related to collagen synthesis. It is characterized by fibroproliferation that mainly affects palmar and digital aponeurosis, causing contractures and progressive flexion deformities. ${ }^{1-3}$ The nodules result from the proliferation of myofibroblasts and accumulation of type III collagen in healthy bands and ligaments composed mainly of type I collagen. Free radicals and ischemia may trigger the disease. It appears to result from a defect in the repair of damaged tissue, an abnormal healing response, an impaired immune response, or a combination of these factors. ${ }^{2-4}$

The incidence ranges from $1 \%$ to $3 \%$, being more common in Northern Europe and more frequent in men than women (6:1). Incidence increases with age, with onset in the fifth decade. ${ }^{1,3,5}$

Sporadic cases exist and are believed to be influenced by a combination of genetic and environmental factors. Some studies suggest environmental influences such as smoking, alcoholism, and diabetes mellitus in particular, as well as other less consistent influences such as HIV, epilepsy/antiepileptic medication and manual work. ${ }^{1,3,4,5}$ Some of these factors are associated with increased free radical release. $^{4}$

Received 27 December 2017.

Accepted 23 February 2018.

* Work conducted at the Dermatology Clinic, Santa Casa de São Paulo, São Paulo (SP), Brazil.

Financial support: None.

Conflict of interest: None.

1 Dermatology Clinic, Santa Casa de São Paulo, São Paulo (SP), Brazil.

2 Division of Internal Medicine, Santa Casa de São Paulo, São Paulo (SP), Brazil.

MAILING AdDRESS:

Karine Simone

E-mail: karine_simone@yahoo.com.br

(C)2018 by Anais Brasileiros de Dermatologia

(cc) BY-NC
The most common location is the palmar region near the fourth, fifth, third and second digits, in this order, in the metacarpophalangeal and proximal interphalangeal joints. ${ }^{1-5}$

The clinical picture is characterized by depressions in the palm, with pigment alteration and fibrosis followed by the onset of generally asymptomatic nodules, corresponding to stage A. In $50 \%$ of the patients, stage A is followed by the formation of cords that cause a progressive retraction, i.e., stage B. Finally, permanent contracture of the fingers, or stage $\mathrm{C}$, can occur. ${ }^{1,3}$

Approximately $5 \%$ of patients have Ledderhose disease, which is associated with plantar fibromatosis, and 3\% have Peyronie's disease, a form of penile fibromatosis. ${ }^{3}$

The physical examination includes the Hueston test, which consists of placing the hand on a flat surface, with fingers extended. The test is positive when the fingers cannot lay flat and instead form an angulation of at least thirty degrees at the metacarpophalangeal joint. No imaging study is recommended; it is necessary only when diagnosis is doubtful. ${ }^{1,4}$ Differential diagnoses are contractures, hyperkeratosis, stenosing tenosynovitis, leiomyoma, and other subcutaneous nodules. ${ }^{1,3}$

In this clinical case, the patient was a 55-year-old female, ex-smoker, with a personal history of discoid lupus erythematosus and diabetes mellitus. She had had an asymptomatic nodule in the left palmar region for 4 months. At the examination, the patient presented a $0.3-\mathrm{cm}$ nodule in the metacarpophalangeal region of the fourth finger, covered by healthy, normochromic and distally retracted skin (Figures 1 and 2). Hueston test was positive. There was no change in function. Based on the hypothesis of Dupuytren's disease, we referred the patient to the orthopedic service.

The treatment of Dupuytren's disease can be divided into non-surgical and surgical. Non-surgical treatment entails clinical observation with annual control in mild, non-progressive and non-limiting cases. An additional possibility is a collagenase injection directly into the pre-tendinous cord, with studies showing complete resolution of the contracture in $57 \%$ to $65 \%$ of cases and recurrence within the first year in $10 \%$ to $50 \%$, being progressive over the years. ${ }^{1,3}$ In early diagnoses, another possible treatment is the injection of triamcinolone; however, a 50\% recurrence rate has been reported in the literature. ${ }^{3,5}$
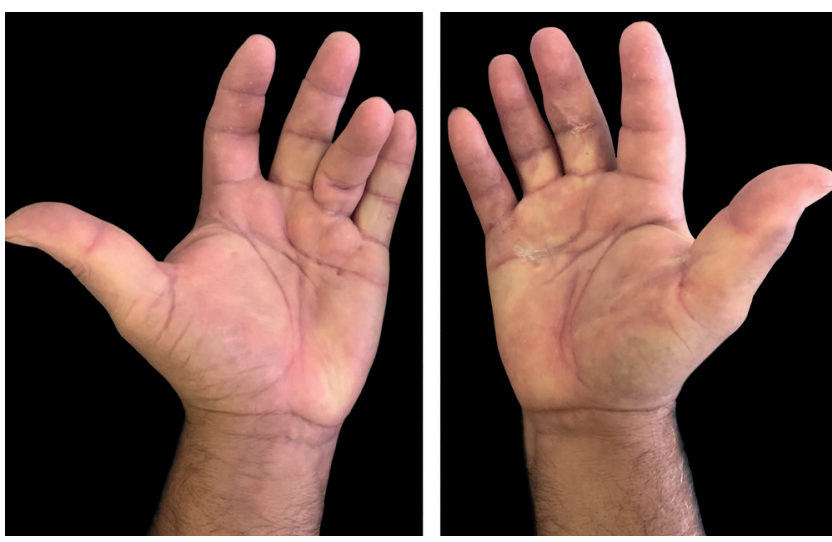

Figure 1: Presence of skin retraction on the left fourth finger 


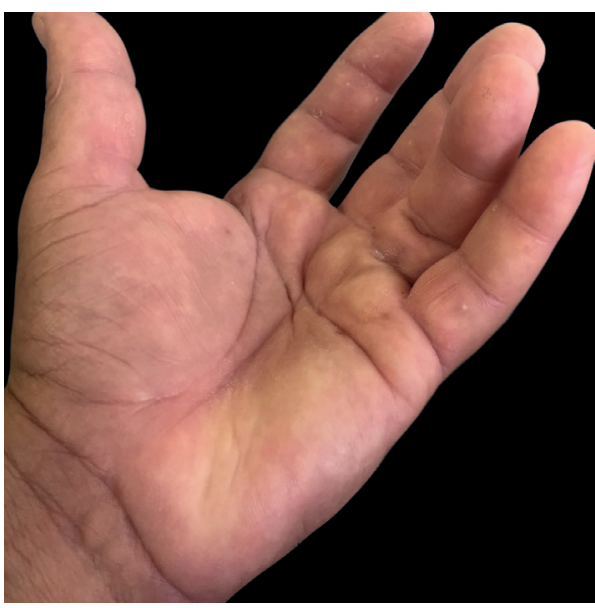

Figure 2:

Nodule in the metacarpophalangeal region of the left fourth finger, covered by healthy skin

\section{AUTHORS' CONTRIBUTIONS}

Camila Ribeiro Amadio

iD ORCID 0000-0001-8980-9815

Approval of the final version of the manuscript, Design and planning of the study, Preparation and writing of the manuscript, Collecting, analysis and interpretation of data, Intellectual participation in propaedeutic and/or therapeutic conduct of studied cases, Critical review of the literature, Critical review of the manuscript

Barbara Machado Magalhães

iD ORCID 0000-0002-4605-9150

Approval of the final version of the manuscript, Design and planning of the study, Preparation and writing of the manuscript, Collecting, analysis and interpretation of data

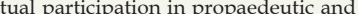
Critical review of the literature, Critical review of the manuscript

Felipe Yazawa $\quad$ (iD) ORCID 0000-0002-7293-4625

Approval of the final version of the manuscript, Design and planning of the study, Preparation and writing of the manuscript, Collecting, analysis and interpretation of data Intellectual participation in propaedeutic and/or therapeutic conduct of studied cases, Critical review of the literature, Critical review of the manuscript

Karine Simone (iD) ORCID 0000-0003-4599-3336

Approval of the final version of the manuscript, Design and planning of the study, Preparation and writing of the manuscript, Collecting, analysis and interpretation of data ration and writing of the manuscript, Collecting, analysis and interpretation of data,
Effective participation in research orientation, Intellectual participation in propaedeutic and/or therapeutic conduct of studied cases, Critical review of the literature, Critical review of the manuscript

For progressive forms of the disease, surgery is the gold standard treatment, indicated for patients with contraction of the metacarpophalangeal joint greater than thirty degrees and contraction of the proximal interphalangeal joint greater than twenty to thirty degrees. ${ }^{1,5}$ Percutaneous fasciotomy, partial open fasciotomy (the most common procedure), total open fasciotomy, dermofasciectomy, segmental aponeurectomy and amputation are all surgical options. ${ }^{1,2,4}$

Male patients have a worse prognosis as well as those that develop the disease before 50 years of age, have Northern European ancestry, family involvement, bilateral impairment, presence of Garrod's nodules and involvement of the proximal interphalangeal joint. The disease may evolve for years and may never cause functional limitation. ${ }^{3}$

In addition to the characteristic clinical picture, our patient had two factors that may contribute to the etiology of the disease: diabetes mellitus and smoking.

Dupuytren's disease is routine for the dermatologist, since the initial presentation of a subcutaneous nodule may be a complaint in a dermatological consultation. It is up to the dermatologist to recognize the disease and its triggering factors, perform early diagnosis, provide family counseling and refer the patient as soon as possible to a specialist for treatment in order to avoid further sequelae.

\section{REFERENCES}

1. Wagner P, Roman JA, Vergara J. Dupuytren disease. Rev Med Chil. 2012;140:1185-90

2. Henry M. Dupuytren's disease: current state of the art. Hand (NY). 2014;9:1-8.

3. Machado S. Doença de Dupuytren: uma visão atual sobre a doença. Rev Port Ortop Traum. 2013;21:313-22.

4. Saraf S. Dupuytren's disease. Indian J Dermatol Venereol Leprol. 2010;76:291-3.

5. Trojian TH, Chu SM. Dupuytren's disease: diagnosis and treatment. Am Fam Physician. 2007;76:86-9.
How to cite this article: Amadio CR, Magalhães BM, Yazawa F, Simone K. Dupuytren's disease: what the dermatologist needs to know. An Bras Dermatol. 2018;93(5):773-4. 